\title{
Dermatologie in Ostafrika
}

\author{
Das Regionale Dermatologische Weiterbildungszentrum (RDTC) \\ in Moshi, Tansania
}

\section{E. Orfanos}

Dermatology in East Africa - The Regional Dermatology Training Centre (RDTC)
in Moshi, Tanzania

Zusammenfassung

Dermatologische Einrichtungen sind in Ostafrika rar, die dermatologische Versorgung ist in Tansania und den angrenzenden Ländern für eine Bevölkerung von insgesamt über 100 Millionen Menschen ausgesprochen mangelhaft. Im Norden Tansanias, in der Provinz um den Kilimandscharo nahe an der Grenze zu Kenia, ist das „Kilimanjaro Christian Medical Center, KCMC“, ein Missionshospital der Good Samaritan Foundation angesiedelt, das auch als Medizinischer Campus der neu gegründeten tansanischen Tumaini Universität dient. Dort ist das „Regional Dermatology Training Center, RDTC" untergebracht, das mit finanzieller Unterstützung der Internationalen Liga gegründet wurde, und unter der Leitung von Prof. Dr. Henning Grossmann und seines Stellvertreters Dr. John Masenga, beide in Deutschland ausgebildete Dermatologen, hervorragende Arbeit leistet. Beide sind in Deutschland ausgebildete, engagierte und erfolgreiche Pioniere, die mit ihrem hohen persönlichen Einsatz die dermatologische Versorgung in Ostafrika gewährleisten.
Abstract

Dermatological institutions are rare in East Africa, and qualified care of patients with skin disease in a population of over 100 million remains clearly insufficient in Tanzania and the neighbouring African countries. In its northern part, in the area of Kilimanjaro close to the Kenyan border, there is the renamed „Kilimanjaro Christian Medical Center", a mission hospital of the Good Samaritan Foundation, also serving as the Medical Campus of the recently founded Tanzanian Tumaini University. Part of the KCMC is the Regional Dermatology Training Center, RDTC, founded with support of the International League and since then led successfully by Prof. Dr. Henning Grossmann and his associate Dr. John Masenga. Both colleagues were trained in Germany, are most engaged and successful physicians, covering important needs for dermatological care of patients in East Africa.
Dermatologische Einrichtungen in Ostafrika sind rar. Während meiner Tätigkeit im Internationalen Komitee der ILDS 1982 - 1997 hatte ich Gelegenheit gehabt, einen Einblick in die dermatologische Versorgung in vielen afrikanischen Ländern zu bekommen und den hohen Bedarf bei der Bevölkerung abzuschätzen. Mit meiner Frau hatte ich zweimal in der Vergangenheit Tansania besucht, und beide Male waren wir von der Schönheit dieses Landes stark beeindruckt. Es waren kurze Aufenthalte, die vor allem mit beruflichen Aufgaben zu tun hatten, dennoch ergab sich immer wieder auch die Chance, das Licht und die Stimmung Afrikas zu spüren, woran bei beiden eine nachhaltige Erinnerung blieb.

So war es seit langem unser Wunsch, wenn ich einmal viel freie Zeit hätte, nach Afrika, vielleicht nach Tansania zu fahren, dort unsere Erfahrungen als Hautärzte zu nutzen, und möglichst länger zu bleiben. Die Zeit fand sich erst nach meiner Emeritierung.

Nach einigen Überlegungen haben meine Frau und ich Anfang vergangenen Jahres den Kontakt mit dem Regional Dermatology 


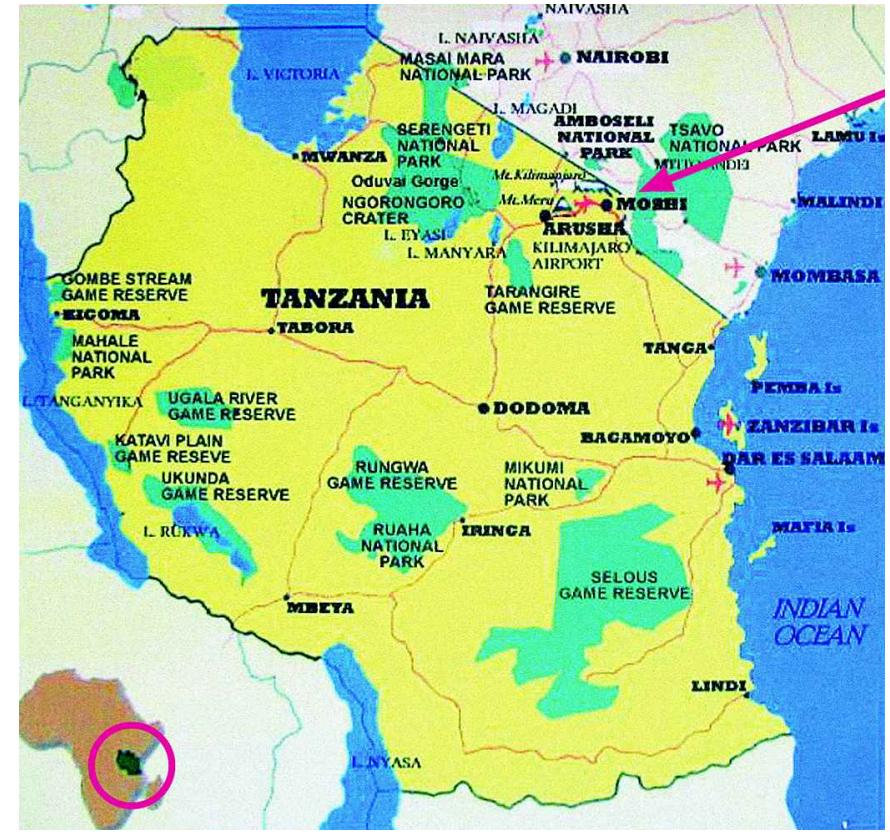

Abb. 1 Lageplan der Kleinstadt Moshi in Tansania, Afrika.

Training Center (RDTC) in Moshi im Norden Tansanias (Abb.1) gesucht, das uns durch vielfältige Kontakte bereits bekannt war. Den Leiter der dortigen Dermatologischen Abteilung, Prof. Dr. Henning Grossmann (Abb. 2), kannte ich bereits seit dem Berliner Weltkongress, und sein Stellvertreter Dr. J. Masenga (Abb. 3) war zunächst DAAD-Stipendiat in meiner Klinik im ehemaligen Klinikum Steglitz der Freien Universität Berlin (jetzt Campus Benjamin Franklin) und hatte gegen Ende der 80er Jahre bei mir die Weiterbildung zum Hautfacharzt absolviert. Er war ein sympathischer junger Kollege und der Kontakt mit ihm hatte die vielen Jahre überdauert.

Mit meiner Frau, ebenfalls Hautärztin, aber im Beruf seit längerem nicht tätig, boten wir an, eine freiwillige, vorübergehende ärztliche Tätigkeit im RDTC aufzunehmen, jeder von uns dort, wo man ihn/sie am ehesten gebrauchen könnte. Herr Prof. Grossmann hat uns in Berlin besucht, unser Vorschlag wurde angenommen, einige Details besprochen und nach einigen Vorbereitungen konnten wir im Januar 2005 das Projekt realisieren. Wir hatten zwar von unserer künftigen Tätigkeit keine genauere Vorstellung, aber wir wussten aus unseren früheren Reisen, dass in den afrikanischen Krankenhäusern ärztliche Hilfe immer benötigt wird, und alles andere würde sich schon finden. Meine Frau hatte einige ihrer Bücher mitgenommen, ich selbst hatte eine Reihe von Vorlesungen vorbereitet und viele notwendige Daten für den Unterricht von Ärzten und Studenten in CDs bzw. in meinem Laptop gespeichert.

Unser Aufenthalt in Moshi/Tansania hat bis Ende März 2005 gedauert, und unsere Erwartungen wurden in jeder Hinsicht bei weitem übertroffen. Als wir nach Berlin zurückkamen hatten wir das Gefühl, viel gegeben, aber noch mehr gewonnen zu haben. In diesem Beitrag wird das RDTC und seine geschichtliche Entwicklung dargestellt, dem wir viel verdanken.

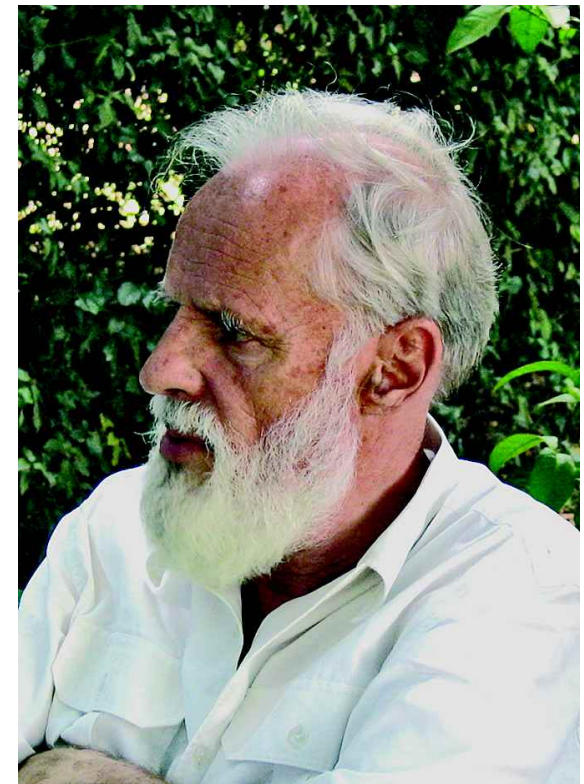

Abb. 2 Prof. Dr. med. Henning Grossmann, Gründer und Prinzipal der Dermatologischen Abteilung des KCMC und Leiter des RDTC.

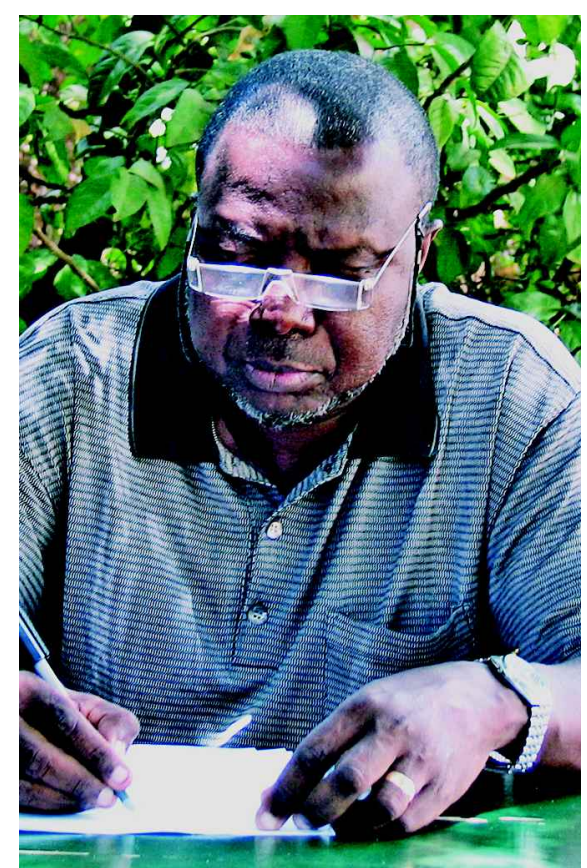

Abb. 3 Dr. med. John Masenga, Stellvertreter von Prof. Grossmann, hat seine dermatologische Weiterbildung in Berlin erfahren und die Facharztprüfung ebendort in deutscher Sprache bestanden.

Das RDTC ist Teil des Kilimanjaro Christian Medical Center (KCMC), das vor 40 Jahren als Missionskrankenhaus an den Hängen dieses mächtigen Bergmassivs, etwa 8 Kilometer außerhalb von der kleinen Provinzstadt Moshi, im Norden Tansanias gegründet wurde (Abb. 4 und 5). Träger ist eine kirchliche Institution, die „Good Samaritan Foundation of Tansania“ (GSF), die dort mit Hilfe einer großzügigen Spende eine alte Kaffee- und Bananenplantage aufkaufte, und Land für den Bau eines Krankenhauses zur Versorgung der Landbevölkerung zur Verfügung stellte. Gründer war Bischof S. R. Moshi und erster Ärztlicher Direktor des neu gegründeten Hauses wurde ein Deutscher, Prof. Dr. Otto Walter.

Tansania ist etwa $2 \frac{1}{2}$ mal so groß wie Deutschland, ein Land das aus der früheren Kolonie Deutsch-Ostafrika bzw. den ehemaligen britischen „Tanganyika territories“ hervorgegangen ist, und 1964 seine Unabhängigkeit erhielt. Der Staat Tansania hat 1971 unter der Präsidentschaft von Julius Nyerere das Missionskran- 


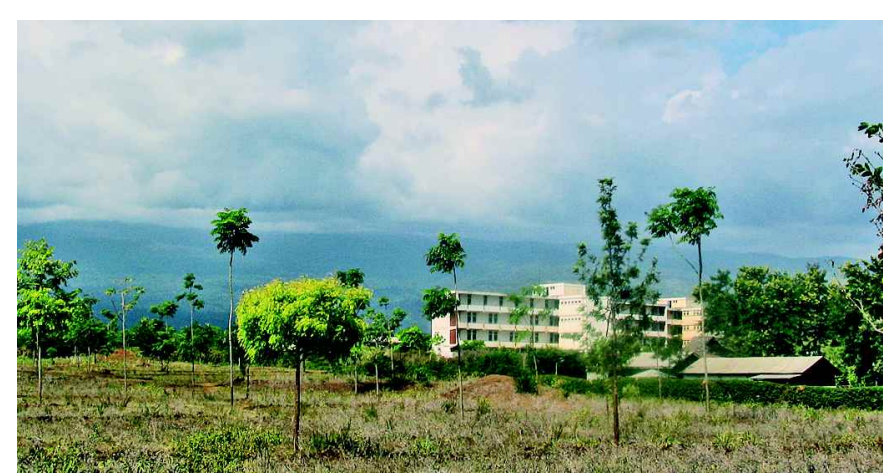

Abb. 4 Das „Kilimanjaro Christian Medical Center“ (KCMC) ist ein modernes Krankenhaus in Moshi.

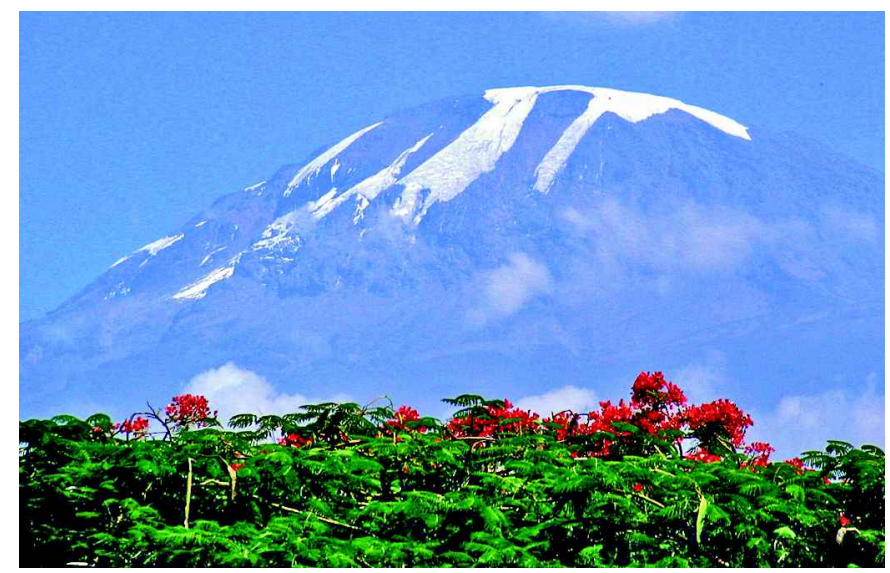

Abb. 5 Der schneebedeckte Kilimandscharo mit 5895 m. ü. Meer der höchste Berg in Afrika ist das Wahrzeichen von Tansania und speziell der Region um Moshi.

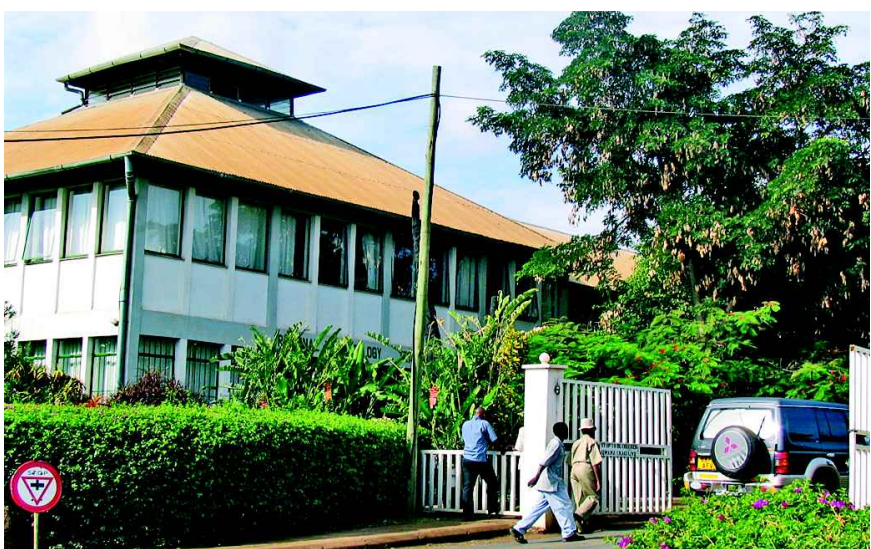

Abb. 6 Das „Regional Dermatology Training Center“ (RDTC) ist Teil des KCMC und verfügt auch über ein Hostel zur Unterbringung der angehenden „Dermatology Officers“.

kenhaus der GSF bei Moshi zunächst verstaatlicht und die Führung selbst übernommen, doch später die Verantwortung erneut an die Kirchenstiftung abgegeben, die die Institution mit staatlicher Unterstützung bis heute trägt und verwaltet. Das Krankenhaus selbst blieb erfreulicherweise von diesen politischen Umwälzungen in seiner Funktion unberührt. Es wurde von der Bevölkerung schnell angenommen, hat während der folgenden Jahre seinen guten Ruf gefestigt, und konnte mit weiteren Bauten ergänzt werden. Bereits 1995 war das KCMC eine der bedeu- tendsten medizinischen Institutionen in Ostafrika, mit fast 90000 ambulanten Patienten, 20000 stationären Aufnahmen und über 3500 Geburten im Jahr. Eine beachtliche Bilanz in relativ kurzer Zeit.

Die Versorgung der Patienten mit Hautkrankheiten und den sexuell übertragbaren Infektionen blieb zunächst anderen, meist den internistischen Abteilungen des Missionskrankenhauses vorbehalten. Erst 1992 wurde innerhalb des Geländes von Dr. Henning Grossmann, einem Dermatologen aus Hamburg mit langjähriger Afrika-Erfahrung, das Regional Dermatology Training Center (RDTC) gegründet (Abb.6). Die dazu notwendigen Mittel mussten freilich anderweitig beschafft werden.

Henning Grossmann gelang es, mit viel Energie und Überzeugungskraft das Internationale Komitee für Dermatologie (ICD), also das oberste Führungsgremium unserer Internationalen Liga Dermatologischer Gesellschaften (ILDS) zu gewinnen, und über eine neu gegründete Stiftung (International Foundation for Dermatology, IFD) eine finanzielle Unterstützung in beachtlicher Höhe zu sichern. Auch ein so genannter internationaler „Club der 500“ wurde ins Leben gerufen, dessen Mitglieder je 500 US Dollar für die Ausstattung des RDTC privat spendeten. Über zweihundert Kollegen aus aller Welt folgten diesem Aufruf, deren Namen heute in einer Ehrentafel im Hauptgebäude des RDTC in Moshi zu lesen sind.

Durch diese Spenden erhielt die Dermatologische Klinik im KCMC einen schönen Neubau mit Ambulanz, Büros, Lehr- und Laborräumen (Abb. 7 und 8). Die feierliche Eröffnung fand am 17. Januar 1997 in Anwesenheit des Ministerpräsidenten der Republik Tansania, F. R. Sumaye, statt, der dafür extra aus Dar es Salaam angereist kam. Mehrere Mitglieder des Präsidiums der ILDS und der IFD waren damals in Moshi anwesend und die Gesamtstimmung war großartig, endlich war das Ziel erreicht.

Einen ersten Anstoß zu einer gedanklichen Neuorientierung der internationalen medizinischen Gremien hatte seinerzeit das ICD-Mitglied Darrell Wilkinson gegeben, der seit langem das ICD drängte, sich verstärkt für eine Verbesserung der dermatologischen Versorgung der Patienten an der Basis zu engagieren, statt Weltkongresse zu organisieren, wo sich schließlich nur Experten untereinander austauschten.

Doch ein weiterer wichtiger Ansatzpunkt, der den Blick für die mancherorts dramatische Unterversorgung der afrikanischen Bevölkerung auf dermato-venerologischem Gebiet schärfte, war die Durchführung und der Erfolg eines Internationalen Workshops mit vielen Vertretern aus Ländern der Dritten Welt, den wir 1987 im Vorfeld des Berliner 17. Dermatologischen Weltkongresses, von unserem Kongress-Sekretariat aus und in enger Zusammenarbeit mit der „Deutschen Stiftung für Internationale Entwicklung“ (DSE) in Berlin auf die Beine gestellt hatten. Soweit ich die Vorgänge vor 20 Jahren noch rekonstruieren kann, kam die Idee von Henning Grossmann. Im Amt des Generalsekretärs des Weltkongresses hatte ich schnell zugesagt. Thema des Vorkongresses war „Dermatology in Basic Health Services“ und Dr. Harald Gollnick, seinerzeit stellvertretender CMD-Sekretär und heutiger Präsident der Deutschen Dermatologischen Gesellschaft, Dr. Henning Grossmann als Experte für die Belange der 


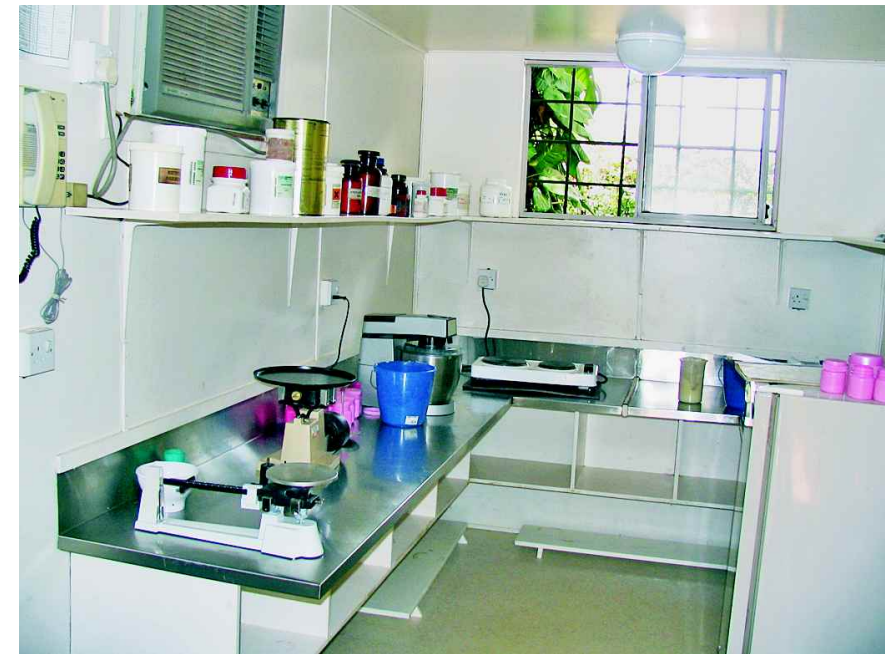

Abb. 7 Das Apothekenlabor im RDTC speziell für die Herstellung von Externa eingerichtet.

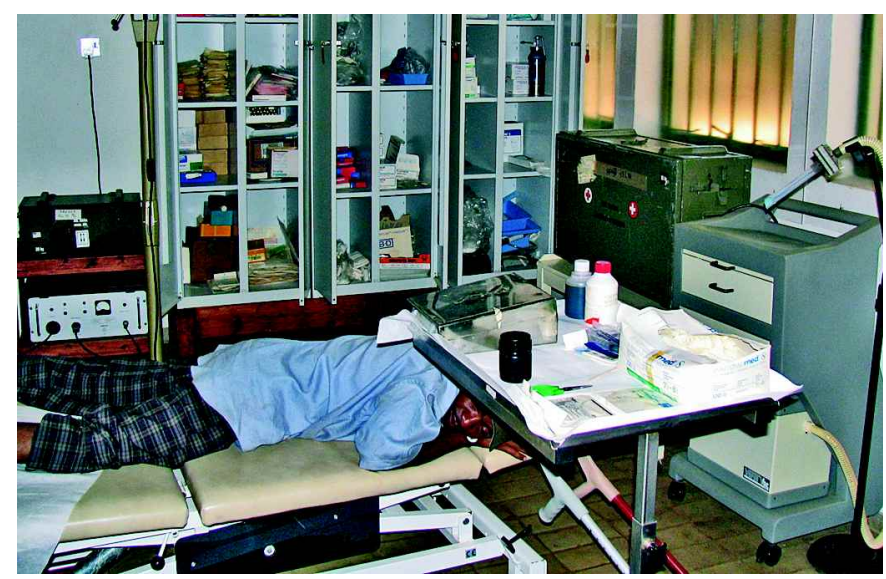

Abb. 8 Der Operationsraum im RDTC.

Dritten Welt und Herr Dr. Dirk Warning als Vertreter der DSE waren damals für die Organisation verantwortlich.

Das Ergebnis übertraf unsere Erwartungen. Immerhin 46 prominente Vertreter aus 26 Entwicklungsländern in allen Kontinenten waren damals nach Berlin gekommen, die sich zum Teil erstmalig begegneten, und fruchtbare Diskussionen auslösten. Das gegenseitige kennenlernen hat allen viel Freude bereitet und Freundschaften sind damals entstanden, die bis heute andauern. Weitere Details dazu sind in zwei Publikationen zu lesen, die nach dem Berliner Kongress erschienen sind [1,2].

Während der Veranstaltungen in der ehemaligen Villa Borsig im Norden des damaligen Berlin-West wurde in eindrucksvoller Weise die dramatische dermatologische Unterversorgung in vielen Ländern der Dritten Welt geschildert und auf die Verantwortung der reichen Länder und der internationalen Organisationen für diese Situation hingewiesen. Auch eine Resolution wurde schließlich verabschiedet, die bei den Delegierten des Berliner Weltkongresses anlässlich der ILDS-Generalversammlung am 27. Mai 1987 große Beachtung fand und vom Plenum einstimmig angenommen wurde.
Darin wurde Folgendes festgehalten:

„The epidemiology of skin diseases in Africa, Asia and Latin America indicates that in general about $30 \%$ of all patients seeking medical help are suffering from skin affections. It appears that a majority of the skin diseases are associated with poor socio-economic conditions. Lack of environmental hygiene, inappropriate housing and environmental conditions favouring parasitic, bacterial and fungal infections. Since no sufficient dermatologists are available - some countries do not have any - the strengthening of dermatology withing primary health care ist clearly needed.“

Und weiter:

„There is need for development of a methodology for rural and peri-urban dermatological care by using reproducible and realistic models. Control of skin diseases, leprosy and sexually transmitted diseases requires the development of inexpensive, appropriate health education materials designed for health personnel of different levels.“

Damit wurde die Stimme der Fachkollegen aus den armen Entwicklungsländern für alle hörbar, und die moralische Verpflichtung westlicher Institutionen, etwas für die unglückliche Situation der Patienten mit Haut- und Geschlechtskrankheiten in den Entwicklungsländern zu unternehmen, wurde während des Berliner Weltkongresses offen diskutiert. Es lag nun nahe, ja es erschien für das in Berlin neu gewählte ICD-Gremium absolut erforderlich, sich für die Dermatologie dort zu engagieren, wo die Armut Hautkrankheiten zu einem gesellschaftlichen Problem machte und eine Beratung und Versorgung auf diesem Gebiet praktisch nicht existent war.

Erstes Ziel des mit Hilfe der jungen Stiftung gegründeten Zentrums war es, möglichst schnell sog. „dermatology officers“ auszubilden, die die primäre dermatologische Versorgung der Bevölkerung in Tansania und den umliegenden Ländern Ostafrikas übernehmen könnten. Es gab auch andere Projekte und Vorschläge, die dem ICD vorlagen, z. B. aus Zentral-Amerika (Guatemala) und auch aus dem frankophonen Teil Afrikas (Mali), doch das Projekt in Tansania bot die meisten Chancen.

Bei den nächsten Sitzungen des ICD wurde beschlossen, ein 2-jähriges dermatologisches Curriculum im Bereich der primären Gesundheitsfürsorge anzubieten, um die dermato-venerologische Beratung und dringliche primäre Versorgung von Patienten mit Haut- und Geschlechtskrankheiten zu sichern. Eine dermatologische Weiterbildung approbierter Ärzte, wie wir sie in Deutschland und anderen europäischen Ländern kennen, und die neben dem vollen Medizinstudium 4- 5 weitere Jahre in Anspruch genommen hätte, schien damals, zumindest in diesem Teil Afrikas, unerreichbar und hätte auch viel zu lange gedauert.

Die Befürworter des Projektes in Ostafrika hatten schließlich im Internationalen Komitee die Mehrheit und das Gremium überzeugt. Auch ein Hostel wurde gestiftet und gebaut, womit die Unterbringung der angehenden „dermatology officers“ im Gelän- 
de des Krankenhauses ermöglicht wurde. Wie wir nachträglich feststellen konnten, war dies, in Anbetracht der lokalen Verhältnisse in Afrika, eine absolut notwendige Einrichtung, um die Unterbringung der Auszubildenden, die aus vielen Ecken des Landes und den angrenzenden afrikanischen Ländern kamen, zu gewährleisten, und den Erfolg des Projektes zu sichern.

Ich kann mich gut an die vielen intensiven Diskussionen während der Sitzungen des ICD, dessen Mitglied ich damals war, über den Sinn und die Notwendigkeit eines langfristigen finanziellen Engagements unseres obersten Gremiums in Ostafrika noch gut erinnern. In der damaligen Satzung der Internationalen Liga Dermatologischer Gesellschaften waren derartige Aktivitäten nicht verankert, und hatten kein Beispiel aus der Vergangenheit.

Schließlich hat das ICD beschlossen, auch die Satzung der ILDS, die mehrere Jahrzehnte nach ihrer Gründung ohnehin einer zeitgemäßen Revision benötigte, entsprechend zu ändern, und in dieser Hinsicht zu vervollständigen. Dies wurde etwas später unter der Präsidentschaft von John Strauss nachgeholt, und in der Vollversammlung der Liga 1992 in New York von den Delegierten genehmigt. In meiner Verantwortlichkeit für die Finanzen des ICD hatte ich fortan die Aufgabe, auch für die regelmäßige finanzielle Unterstützung unserer Foundation von Seiten der Liga zu sorgen, die zu einem großen Teil für das Zentrum in Moshi gedacht waren.

Bei einem Besuch im Jahre 2001 stellten wir mit dem ICD zu unserer großen Freude fest, dass diese Gelder zweifelsohne sehr gut angelegt worden waren.

Das von der Stiftung der ILDS mitgetragene RDTC wurde die Dermatologische Abteilung des Missionskrankenhauses am Kilimanjaro, mit einer beachtlichen Ambulanz und eine Bettenstation von bis zu 25 Betten im Hauptgebäude des Klinikums. Prof. Dr. Henning Grossmann wurde als Leiter (Principal) der Abteilung eingesetzt. Das von ihm initiierte und propagierte Vorgehen zur Ausbildung diplomierter „dermatology officers“ wurde mit Erfolg gekrönt, zumal es ihm wenige Jahre später gelang, diesen Lehrgang im offiziellen Lehrprogramm der University of Dar es Salaam Medical School zu verankern.

Über 140 Diplomanden in Dermato-Venerologie wurden inzwischen vom RDTC in Tansania ausgebildet und versorgen heute in über 15 verschiedenen afrikanischen Ländern Patienten mit Haut- und sexuell übertragbaren Krankheiten, die in Afrika alles andere als selten sind. Prof. Grossmann hat inzwischen als Arzt und Principal im RDTC einen national und international hervorragenden Ruf, er ist eine hochgeschätzte Persönlichkeit und gesuchter Diskussionspartner.

Es war ein glücklicher Umstand, dass im Jahre 1998 das KCMC, dessen Bedeutung für den gesamten ostafrikanischen Bereich unübersehbar wuchs, zum Medical College der Medizinischen Fakultät der 1993 neu in Tansania gegründeten staatlichen $\mathrm{Tu}$ maini University wurde. Somit erhielt die dermatologische Abteilung universitären Status und hat die Aufgabe übernommen, Studenten der Medizin im Pflichtfach Dermato-Venerologie zu unterrichten. Vor nicht allzu langer Zeit kam vom Gesundheitsmi-

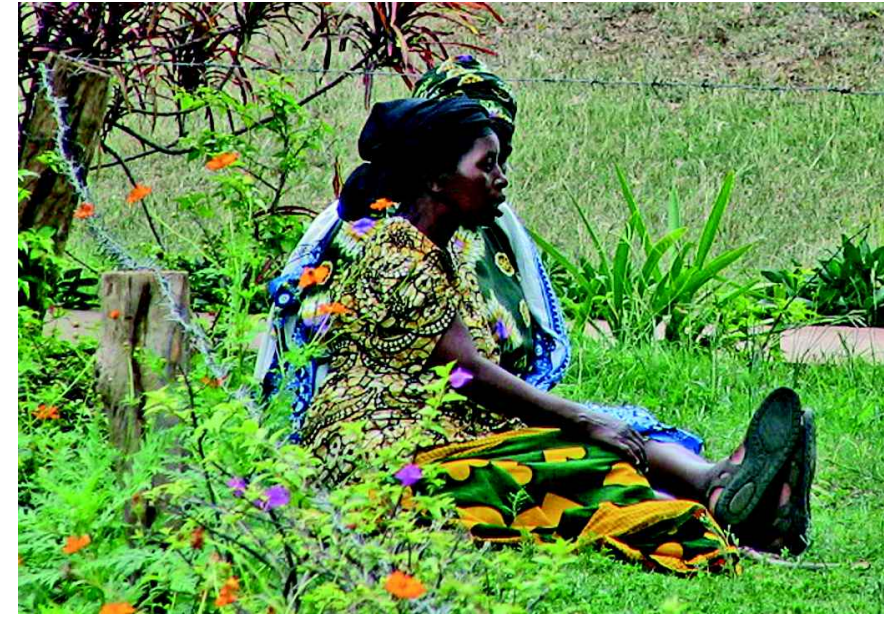

Abb. 9 Wartende Patienten im Gelände des KCMC.

nisterium auch die Berechtigung für die volle 4-jährige Weiterbildung im Fach Dermato-Venerologie, ein Vorgang der für Tansania und ganz Ostafrika von außerordentlicher Bedeutung ist.

In Tansania gibt es kaum Hautfachärzte, sie sind mit den Fingern einer Hand zu zählen. Im Ostteil des Teils des afrikanischen Kontinents gab es bisher keine einzige Einrichtung, die für die Weiterbildung von Ärzten auf dem Gebiet der Haut- und sexuell übertragbaren Krankheiten zugelassen ist, weder in Tansania noch im benachbarten Kenia oder in Äthiopien, geschweige denn in Erythrea oder in Mosambique, d.h. in einem Gebiet mit weit über 100 Millionen Menschen! Dabei sind es gerade Hautfachärzte, die mit den Geschlechtskrankheiten vertraut sind, die man heute braucht, um die Bevölkerung über die sexuelle Übertragung solcher Infektionen aufzuklären, sie zu betreuen, und nicht zuletzt auch die HIV- und AIDS-Epidemie wirksam zu bekämpfen.

Trotz dieser Erfolge, die in vollem Umfang auf die unermüdliche Arbeit von Henning Grossmann und seines unmittelbaren Vertreters und engen Mitarbeiters, John Masenga, zurückzuführen sind, ist die Versorgung dermatologischer Patienten in den Ländern Ostafrikas immer noch sehr unbefriedigend (Abb. 9). Dabei hat gerade heute die Dermatologie eine herausragende Bedeutung für die gesundheitliche Situation in allen Ländern des afrikanischen Kontinents. Sie ist für die primäre Versorgung überaus wichtig, nicht zuletzt durch ihre Verknüpfung mit den sexuell übertragbaren Krankheiten, ihre engen Beziehungen zu HIV-Infektion und AIDS, aber auch durch die vielen tropischen Infektionen, die sich häufig an der Haut manifestieren. Dazu gehören die Lepra, die Onchozerkose, die Filariosen, Leishmaniose, Schistosomiasis, die tropischen Ulzera und viele andere.

Ein langjähriges Festhalten an völlig ungeeigneten ärztlichen Aus- und Weiterbildungsmodellen aus der kolonialen Tradition mancher afrikanischer Länder, ebenso wie menschliches Versagen, waren und sind noch für diese Situation verantwortlich. Mit gutem Grund kann man sie dramatisch nennen.

Hier sollte man anführen, dass neben Prof. Dr. Grossmann auch Dr. Masenga den Titel eines deutschen Facharztes führt. Letzterer 
hat immerhin als erster Afrikaner an der Freien Universität in Berlin seine mündliche Facharztprüfung (auf Deutsch) vor über 15 Jahren mit Erfolg bestanden. Unter der persönlichen Leitung von Dr. Masenga, der in seiner Heimatstadt Moshi mit seiner Familie hohes Ansehen genießt, steht die Venerologie sowie die Betreuung der HIV-Patienten, ein Brennpunkt der medizinischen Versorgung. Speziell für die sexuell übertragbaren Infektionen wird von ihm in der Stadt eine Beratungs- und Behandlungsstelle geführt und als Außenstelle des RDTC betrieben. Er hat beste Chancen die Nachfolge des Visionärs Grossmann anzutreten.

Henning und John, zwei seit vielen Jahren in Ostafrika tätige, in Deutschland gut ausgebildete Hautfachärzte, haben inzwischen enormes geleistet und verdienen für ihren langjährigen und unermüdlichen Einsatz für die vielen kranken Menschen und für das Fach höchste Anerkennung.

Die Grossmanns leben seit fast 25 Jahren in Tansania. Die Familie ist heute fest in Moshi angesiedelt und der jüngste Sohn studiert im 4. Jahr Medizin. Immerhin, er ist der erste weiße Medizinstudent der neu gegründeten tansanischen Tumaini Universität! Frau Herma Grossmann ist mit vielen sozialen Projekten in der Stadt beschäftigt und gilt als die Muse und die gute Seele der Familie. Die Grossmanns werden von allen Patienten selbstverständlich als Tansanier angesehen, zumal sie alle gut die Landessprache Kiswahili sprechen.

Mit seiner gewinnenden Persönlichkeit, die die Aura eines Albert Schweitzer ausstrahlt, ist Dr. Grossmann allgegenwärtig im RDTC. Neben seinen Engagements in zahlreichen Kommissionen und Projekten, die Fertigstellung der diversen Lehrpläne für Medizinstudenten, die Ausbildungsprogramme und Prüfungen für die „dermatology officers“ und nicht zuletzt die dermatologische Weiterbildung der jungen Ärzte in seiner Abteilung, ist er ständig darum bemüht, Sponsoren zu suchen, um die finanzielle Unabhängigkeit des RDTC, auf die er mit Recht stolz ist, zu erhalten und auch für die Zukunft sicherzustellen.

Natürlich muss er bei den Verhandlungen mit Lieferanten von wichtigen Medikamenten aus Indien selbst dabei sein, wenn es darum geht, den Preis für eine 4-wöchige Behandlung der HIVInfektion von 32000 auf 18000 TS (ca.18 US Dollar) herunter zu drücken. Am liebsten würde er die Medikamente als Industrie- spende ohne jedes Entgelt für seine kranken Patienten haben wollen!

Die Zuschüsse der tansanischen Regierung wären ohne die zusätzlichen Sponsorengelder, die mit großem Geschick und Ausdauer über die Jahre angeschafft werden müssen, bei weitem nicht genug, um die dringendsten Aufgaben in der Versorgung der vielen bedürftigen Patienten zu bewältigen. Dazu gehörten Spenden an Geld und an Medikamenten, Ausstattung für die Büros sowie Apparaturen und Materialien für wichtige Laboruntersuchungen.

Das RDTC ist nun durch diese Aktivitäten verhältnismäßig gut ausgestattet und gerade Gastärzte, die immer wieder vorübergehend im Zentrum tätig sind, wissen das zu schätzen. Dr. Masenga ist über die Jahre an der Seite von Henning bestens gereift und hat ihm im Lehrprogramm und im besonders wichtigen klinischen Bereich der Venerologie und HIV-Infektion den Rücken gestärkt. Henning und John sind ein erfolgreiches Gespann.

Die Dermatologie bzw. die Versorgung von Patienten mit Hautund sexuell übertragbaren Krankheiten ist im ostafrikanischen Raum durch die Gründung und die Tätigkeit des RDTC am Kilimanjaro in Moshi ein großes Stück weiter gekommen. Es ist zwar noch sehr viel zu tun, doch eine funktionsfähige Basis ist inzwischen vorhanden, wovon viele bedürftige kranke Menschen profitieren. Dies verdanken wir und die Dermatologie ohne jeden Zweifel der unermüdlichen Arbeit zweier in Deutschland ausgebildeter Ärzte und Pioniere im Fach, die sich seit vielen Jahren mit voller Kraft für ihre Patienten einsetzen.

Während unseres Aufenthaltes konnten wir ein wenig dazu beitragen, und haben selbst viel gelernt. Es sind unschätzbare fachliche und menschliche Erfahrungen, die uns bereichert haben.

\section{Literatur}

${ }^{1}$ Grossmann H, Warning D (eds). Dermatology in Basic Health Services. Proceedings of the International Workshop held in Berlin (West) on 20-23 May 1987. Deutsche Stiftung für Internationale Entwicklung (Publisher) ISBN 3-924441-35-9, 1988

2 Orfanos CE, Stadler R, Gollnick H, Garbe C (eds). Der Berliner Kongress. Daten - Analysen - Echo der Presse. Berlin: Grosse Verlag ISBN 3-88040-064-4, 1988 